Provided for non-commercial research and education use. Not for reproduction, distribution or commercial use.

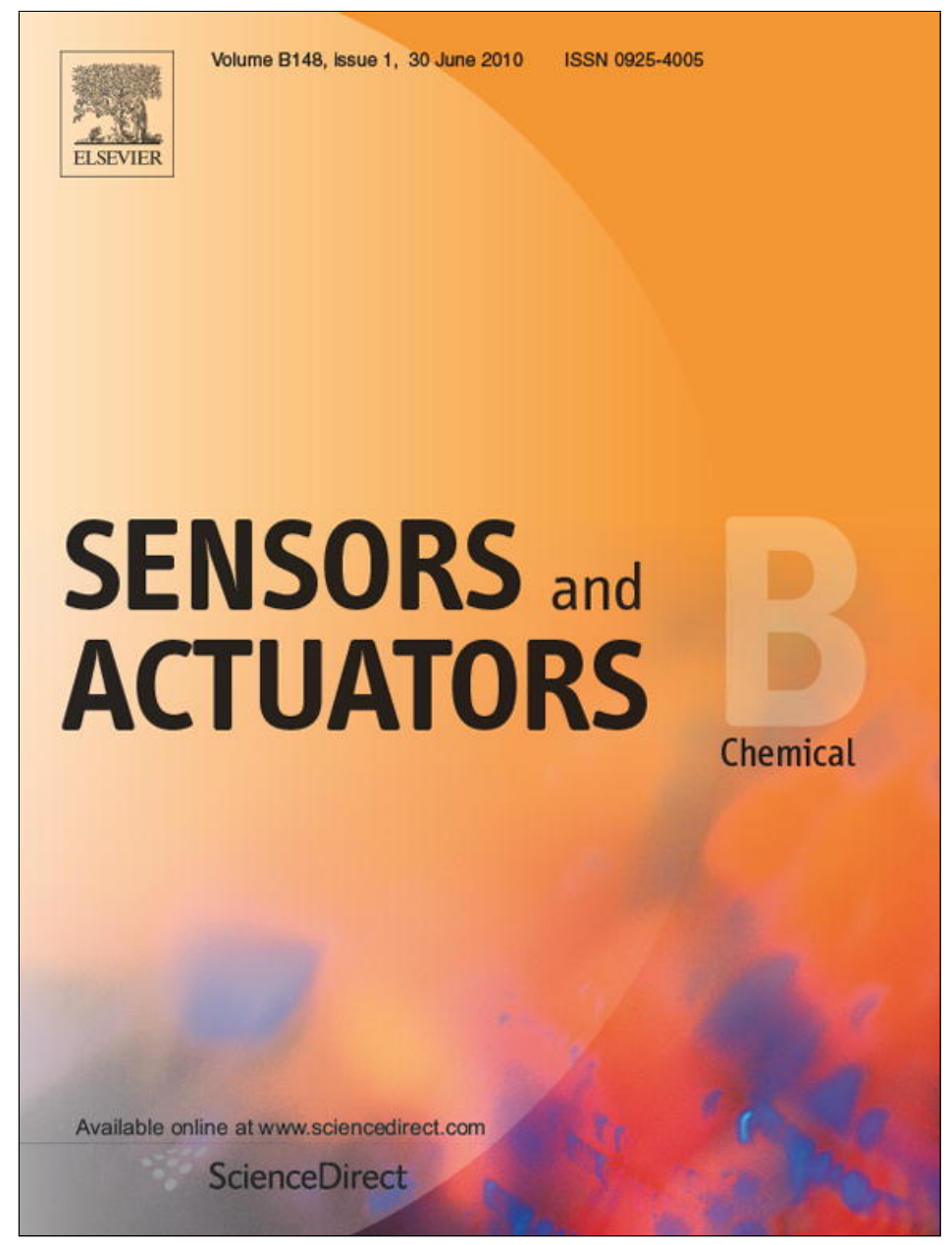

This article appeared in a journal published by Elsevier. The attached copy is furnished to the author for internal non-commercial research and education use, including for instruction at the authors institution and sharing with colleagues.

Other uses, including reproduction and distribution, or selling or licensing copies, or posting to personal, institutional or third party websites are prohibited.

In most cases authors are permitted to post their version of the article (e.g. in Word or Tex form) to their personal website or institutional repository. Authors requiring further information regarding Elsevier's archiving and manuscript policies are encouraged to visit:

http://www.elsevier.com/copyright 


\title{
Chemical sensing using a polymer coated long-period fiber grating interrogated by ring-down spectroscopy
}

\author{
J.A. Barnes, R.S. Brown, A.H. Cheung, M.A. Dreher, G. Mackey, H.-P. Loock* \\ Department of Chemistry, Queen's University, 90 Bader Lane, Kingston, ON, K7L 3N6, Canada
}

\section{A R T I C L E I N F O}

Article history:

Received 25 January 2010

Received in revised form 30 March 2010

Accepted 5 April 2010

Available online 24 April 2010

\section{Keywords:}

Long-period grating

Fiber optic sensor

Cavity ring-down

Refractive index sensing

Solid-phase microextraction

\begin{abstract}
A B S T R A C T
An etched long-period grating was used as a refractive index sensor for vapours of four volatile organic compounds, i.e. m-xylene, cyclohexane, trichloroethylene and commercial gasoline. The sensitivity to the vapours was further increased by solid-phase microextraction into a coating made of polydimethylsiloxane (PDMS)/polymethyl-octylsiloxane (PMOS) co-polymer. By further amplification of the optical loss in an optical cavity made of two identical fiber-Bragg gratings and interrogation by phase-shift cavity ring-down spectroscopy we could detect and distinguish xylene (detection limit: 134 ppm) from trichloroethylene (3300 ppm), cyclohexane (1850 ppm) and gasoline (10,500 ppm).
\end{abstract}

(C) 2010 Elsevier B.V. All rights reserved.

\section{Introduction}

For about a decade, long-period gratings (LPGs) have been used as sensor elements to measure refractive index (RI) of liquids and films [1-5]. LPGs are periodic perturbations of the RI in the core of a single mode optical fiber with a periodicity typically between $\Lambda=200$ and $500 \mu \mathrm{m}$. The transmission spectrum of an LPG shows attenuation features at wavelengths given by the phase matching condition:

$\lambda_{i}=\Lambda\left[n_{\text {core }}^{\text {eff }}\left(\lambda, n_{\text {core }}, n_{\text {clad }}\right)-n_{\text {clad }, i}^{\text {eff }}\left(\lambda, n_{\text {core }}, n_{\text {clad }}, n_{a}\right)\right]$

where $n^{e f f}$ is the effective RI and $n_{a}$ is the ambient RI. An attenuation feature occurs when the core mode is coupled into a co-propagating cladding mode, with an effective RI that depends on the RIs of the core, cladding and environment. Light propagating in the cladding is then dissipated, which leads to a drop in fiber transmission. Any physical parameter that affects either the grating's periodicity or the effective RI of the fiber core or cladding will lead to a shift in $\lambda_{i}$, thus making LPGs sensitive to ambient RI, temperature, strain, etc.

Chemical sensing using an LPG may be accomplished by exploiting the ambient RI sensitivity of the grating resonances. Chemical selectivity can be achieved by application of coatings to the grating that display selective chemical adsorption. For example, functionalization of the fiber surface can provide selective and strong binding

\footnotetext{
* Corresponding author. Tel.: +1 6135332621; fax: +1 6135336669.

E-mail address: hploock@chem.queensu.ca (H.-P. Loock).
}

to the analyte of interest [6-8]. Alternatively, a thick (compared to the depth of the evanescent wave) coating can be deposited on the fiber surface and solid-phase microextraction (SPME) may be used to select and preconcentrate analytes of interest while also increasing the sensitivity of the LPG by tuning the RI of the coating $[9,10]$. A moderate chemical selectivity can again be achieved by chemical modification of the coatings.

Absorption of an analyte into a polymer coating on the LPG increases or decreases the coating's RI, and shifts the position of the LPG resonance to shorter or longer wavelength, respectively. The magnitude of this shift depends not only on the RI of the analyte and its partitioning coefficient but also on the initial RI of the polymer coating. A strong response is expected when the coating's $\mathrm{RI}$ is slightly lower than that of the fiber's cladding material.

In this report, the response of the LPG was further amplified by placing it into an optical cavity, which was formed by two identical fiber-Bragg gratings (FBGs). We then used phase-shift waveguide cavity ring-down (CRD) spectroscopy to monitor the LPG attenuation at a fixed wavelength given by the FBG's reflection band. CRD absorption spectroscopy has the advantage that the loss inside an optical cavity is obtained from a photon lifetime measurement and that this "ring-down time", $\tau$, is independent of the light intensity and is immune to detector drifts [11]. CRD spectroscopy is also very sensitive since the sensing element interacts with the trapped light pulse many times and the optical loss is accordingly amplified by the cavity gain factor (typically $10^{2}$ to $10^{4}$ ) [12]. Instead of the more commonly used time-domain measurements of ringdown times we employed the phase-shift CRD technique, since it is simple to implement, has a much greater duty cycle and is fully 


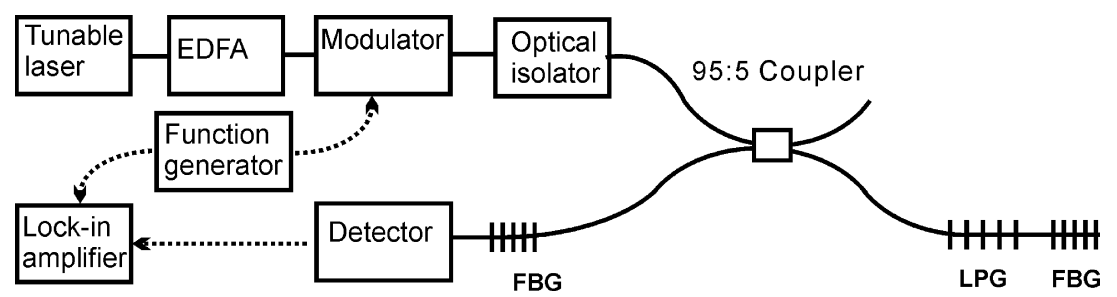

Fig. 1. Scheme of the experimental setup.

compatible with inexpensive light sources and detectors [13]. In phase-shift CRD intensity-modulated continuous-wave laser light is injected into the loop. Light exiting the loop is shifted in phase with respect to the input phase by virtue of the optical lifetime of light captured within the optical cavity. The optical lifetime of the cavity, $\tau$, is determined from the phase-shift, $\Delta \Phi$, through the relationship $\tan \Delta \Phi=-\Omega \tau$, where $\Omega$ is the modulation frequency. A change in the measured phase-shift, $\Delta \Phi$ is caused by changes in loss within the loop, which are created by absorption of chemicals into the coating and the consequent shift in the attenuation peak of the LPG with respect to the laser wavelength.

In a previous report, the optical cavity was formed from a loop of optical fiber; an LPG was placed in the loop, coated with polydimethylsiloxane based polymer, and its response to a volatile aromatic compound (xylene) and a simple hydrocarbon (cyclohexane) was characterized [14]. The present work employs the same CRD techniques, but rather than using a loop of optical fiber, a linear cavity is formed using two identical fiber-Bragg gratings (FBGs) as reflective elements. Liu et al. previously described a similar setup consisting of an FBG cavity, which contained an LPG [15]. In their experiment the LPG was used for strain and temperature sensing and, as in our case, the ring-down time of the cavity was determined. The differences to Liu's previous work are, first, in the CRD techniques - we use SPME and phase-shift CRD measurements and, second, in the application, i.e. chemical sensing of a variety of organic vapours.

\section{Preparation of the sensor system}

\subsection{Cavity ring-down system}

The optical cavity consisted of two FBGs (ITF Labs, Montréal, QC, Canada) with reflectivities $>99.9 \%$ and a bandwidth of $0.36 \mathrm{~nm}$ connected by $6 \mathrm{~m}$ of Corning SMF-28 single mode optical fiber. The center wavelengths for the FBGs were matched at $1550.07 \mathrm{~nm}$. Contained within the cavity was an LPG (ITF Labs) having a periodicity of $236 \mu \mathrm{m}$, a length of $22 \mathrm{~mm}$, and a maximum attenuation of $-1.3 \mathrm{~dB}$. Light was injected into the cavity using a 95:5 $2 \times 2$ directional coupler (Lightel Technologies Inc., South Kent, WA) positioned between the FBGs (Fig. 1). An InGaAs detector (Model PDA10CS, Thorlabs Inc., Newton, NJ) was placed outside the optical cavity, behind one of the FBGs, to measure the cavity transmission. From the ring-down time of $137 \mathrm{~ns}$ (see below) the total cavity roundtrip loss was determined to be $20 \%$. The loss is only weakly dependent on the reflectivity of the FBGs, but rather is dominated by the three fiber splices in the cavity with an estimated combined loss of less than 5\%, the insertion loss of the LPG (about $5 \%$ ) and the loss at the coupler $(>10 \%)$.

The phase angle of the amplified detector signal was determined using a lock-in amplifier (SR844, Stanford Research Systems, Sunnyvale, CA). A data acquisition card (PMD-1608FS, Measurement Computing, Middleboro, MA) transferred the lock-in output to a computer for analysis.

A tunable diode laser (AQ4320D, ANDO, Yokogawa Electric Corp., Tokyo, Japan) set at $1550.07 \mathrm{~nm}$, was used to excite the optical cavity. The laser had an output power of $3.5 \mathrm{~mW}$ with a measured linewidth of $165 \mathrm{MHz}$ (measured using an Agilent 83453A High Resolution Spectrometer). Due to the low transmission of the FBGs, it was necessary to amplify the diode laser output before injection into the optical cavity. This was done using an erbium doped fiber amplifier (Model OIBTC-18-SdBGPFA, Keopsys, Lannion, France). The amplified output power was approximately $30 \mathrm{~mW}$. Intensity modulation was achieved by passing the EDFA output through a Mach-Zehnder modulator (JDS Uniphase) modulated by a function generator (Dae Shin DOA-141, Rotronic, Switzerland). A dual stage optical isolator (Lightel Technology) was used between the modulator and the input coupler to reduce optical feedback. Optical feedback was further reduced by coating the unused connections on the $2 \times 2$ coupler and FBG with index matching gel.

\subsection{Polymer-coated LPG}

As noted above, the position of the resonance peak of an LPG depends on the ambient refractive index adjacent to the grating. The sensitivity of the attenuation peak's wavelength to the ambient RI change, $d \lambda_{i} / d n_{a}$, is not a constant, but rather increases dramatically as $n_{a}$ approaches $n_{\text {clad }}$. Also, $d \lambda_{i} / d n_{a}$ increases with increasing cladding mode order, $i[1]$. Sensitivity to perturbations in ambient refractive index will therefore be enhanced when working close to $n_{\text {clad }}$ and when observing the response of a high-order cladding mode. For SMF-28 fiber, $n_{\text {clad }}=1.4453$ at $1550 \mathrm{~nm}$.

The response of an isolated LPG, which was not yet inserted into a cavity, was observed using an optical spectrum analyzer (Agilent 86142B, Santa Clara, CA). An LPG with comparatively short period, $\Lambda=236 \mu \mathrm{m}$, was chosen because it places a high-order cladding mode $\left(\mathrm{LP}_{09}\right)$ within the scan range of the OSA and the FBG cavity. As provided, the attenuation due to coupling into the $\mathrm{LP}_{09}$ cladding mode of the grating appears at $1410 \mathrm{~nm}$. When coated with polymer, the mid-point of the attenuation peak must appear in the vicinity of $1550 \mathrm{~nm}$ so that it overlaps the bandwidth of the FBG cavity. To achieve a final mode position near $1550 \mathrm{~nm}$ after polymer coating, it is necessary to first shift the mode position of the uncoated LPG to even longer wavelength, so that the attenuation peak will blue-shift to $1550 \mathrm{~nm}$ when the coating is applied and condensed. A polymer target refractive index value of 1.43 was chosen. Cladding modes may be shifted to longer wavelength by reduction in the cladding diameter through chemical etching. As described elsewhere, a saturated aqueous solution of ammonium bifluoride was used to etch the LPG [14]. The etching process was monitored by observing the transmission spectrum of the grating. During the etching process, the etching solution was removed periodically and replaced by a $77 \%$ dimethyl sulfoxide/water solution, which has a refractive index of 1.4303 at $1550 \mathrm{~nm}$. Etching was continued until immersion in the DMSO solution resulted in a mode position that overlapped the cavity bandwidth. Fig. 2 shows the change in peak position of the etched LPG with changing ambient refractive index for values near the polymer target value. The average rate of change in this region is $d \lambda_{i} / d n_{a}=-41,000 \mathrm{~nm} / \mathrm{RIU}$. This sensitivity to refractive index is $1-2$ orders of magnitude 


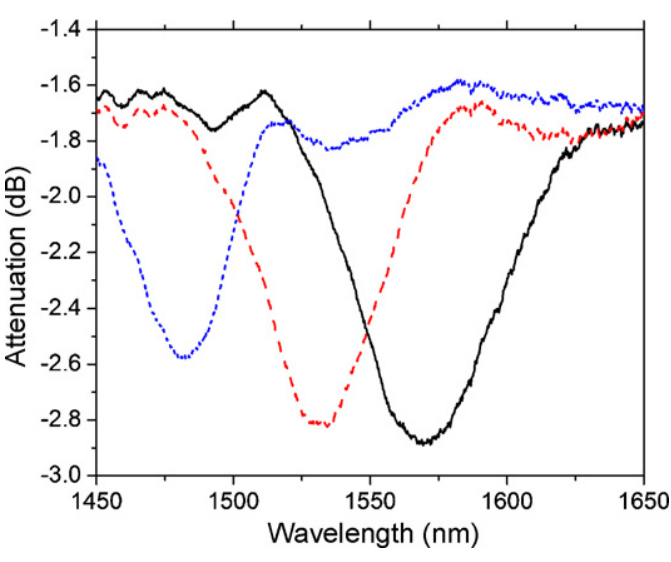

Fig. 2. Response of the etched and uncoated LPG to three water DMSO solutions containing 77.5\% DMSO (black, solid), 78.5\% DMSO (red, dashed), and 79.5\% DMSO (blue, short dashed). The RIs increase by $1.1 \times 10^{-3}$ with each $1 \%$ increase in DMSO concentration. The sensitivity of the RI measurement is therefore about $41,000 \mathrm{~nm} / \mathrm{RIU}$. (For interpretation of the references to color in this figure legend, the reader is referred to the web version of the article.)

higher compared to what is usually observed with off-the-shelf LPGs.

A derivative of polydimethyl siloxane (PDMS) was then used to coat the LPG. Pure PDMS, a material commonly used in SPME, has a refractive index of 1.36 , which is much too low for our needs. The refractive index of the polymer coating was therefore adjusted by forming a 7:13 co-polymer of dimethyl siloxane and methyl-octyl siloxane and by cross-linking with 3\% titanium tetraisopropoxide. Details of the synthesis and characterization of the PDMS/PMOS co-polymer are described elsewhere [16]. The refractive index of the co-polymer was $1.4278 \pm 0.0005$ at $1550 \mathrm{~nm}$ and was measured using a calibrated Abbé refractometer that was built in-house. The refractometer used the collimated output of a $1550 \mathrm{~nm}$ laser diode and a NIR-sensitive CCD camera. This polymer coating was applied to the LPG using a 5:1 dilution of the appropriate pre-condensed siloxane oligomers and cross-linker in hexane. A droplet of the hexane solution was repeatedly pulled along the length of the LPG until the observed peak position shift remained constant. The coated LPG was then cured in an oven at $80^{\circ} \mathrm{C}$ for $110 \mathrm{~h}$. A transmission spectrum of the FBG cavity, containing the polymer-coated LPG, is shown in Fig. 3.

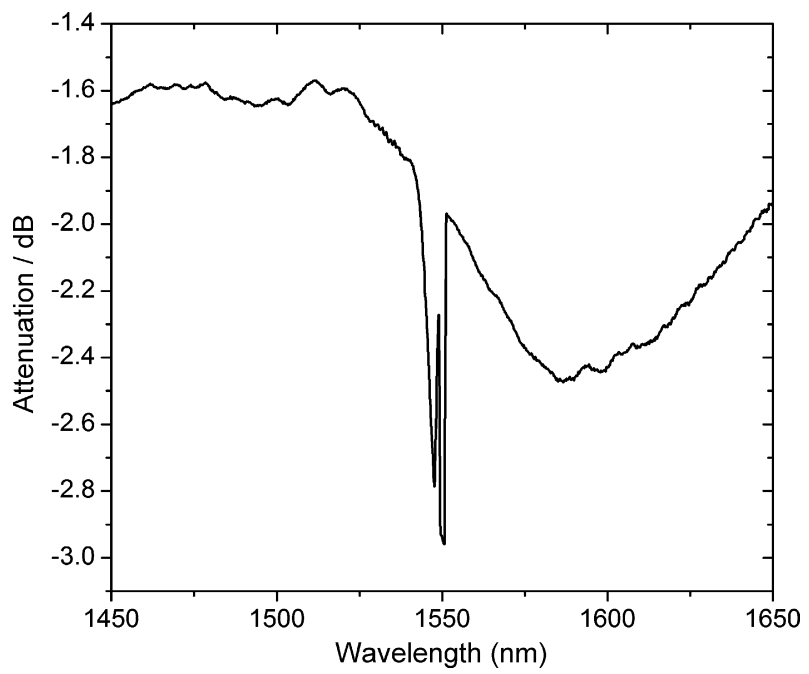

Fig. 3. Transmission spectrum of the FBG cavity containing the coated LPG in $100 \%$ nitrogen gas.
A fixture was designed to allow the polymer-coated LPG to be suspended within a closed compartment, and to expose it to a flowing gas stream containing the analyte vapour. The vapour stream was created by bubbling nitrogen through a gas washing bottle containing pure solvent. The vapour stream was combined with pure nitrogen in a mixing flow-meter to adjust the concentration. The gas mixture from the flow-meter passed through a Teflon ${ }^{\circledR}$ tube to the inlet of the cavity surrounding the LPG. The cavity volume was $2.2 \mathrm{~cm}^{3}$ and the net gas flow rate was maintained at $88 \mathrm{~cm}^{3} \mathrm{~min}^{-1}$.

A similar apparatus was used in liquid detection. A Teflon ${ }^{\circledR}$ well with a volume of $2.2 \mathrm{~cm}^{3}$ was completely filled with a m-xylene water solution and subsequently covered to prevent the analyte from equilibrating with the air. The aqueous solution was prepared by adding $\mathrm{m}$-xylene to water, followed by prolonged mixing with a stir bar. From this, a 1:1 dilution of the m-xylene saturated water was made.

\section{Results and discussion}

After the LPG was spliced into the cavity but before it was coated with PDMS/PMOS polymer, the $\mathrm{LP}_{09}$ attenuation peak was outside the detection range. The optical lifetime of the cavity was then determined by measuring the phase-shift of the cavity transmission as a function of modulation frequency. We expect, for a single exponential decay process, a linear dependence of $\tan \left(\Phi-\Phi_{0}\right)$ on the angular modulation frequency, $\Omega$, with a slope given by the negative cavity lifetime, $\tau[131$. Here $\Phi(\Omega)$ is the measured phase angle and $\Phi_{0}(\Omega)$ is a frequency-dependent instrumental offset due to propagation delays, electronic time constants, etc. If multiple decay processes are present, fitting to a more complicated functional form allows for extraction of the individual ring-down times and the associated relative decay amplitudes [17]. The offset value, $\Phi_{0}(\Omega)$, was measured by tuning the laser to a wavelength $(1545 \mathrm{~nm})$ well outside the FBG bandwidth and injecting the laser into the cavity in the direction of the detector.

Diode lasers typically emit a small fraction of their output as amplified spontaneous emission (ASE), which has a much broader frequency distribution than the stimulated emission. As a consequence, the majority of the ASE will lie outside the bandwidth of the FBGs and thus make only a single pass through the cavity. If ASE is not suppressed, the phase angle data will indicate the presence of two lifetimes. One lifetime will be very short, corresponding to ASE traversing the cavity, and the other, longer lifetime results from light trapped in the FBG cavity [18]. In this experiment ASE was easily suppressed by injecting the laser into the cavity such that it must reflect from a FBG before reaching the detector. The results of the ring-down time measurement with the uncoated LPG in the FBG cavity but outside the detection range are shown in Fig. 4. A single time constant, having a value of $\tau=137 \mathrm{~ns}$, describes the data. This indicates that ASE has been successfully suppressed.

From the ring-down time and the cavity length, $L=6 \mathrm{~m}$, the effective number of times the LPG interacts with a "trapped photon" can be calculated as $\tau c_{0} / n L=4$.7, where the refractive index $n \approx 1.46$ for the core mode and $c_{0}$ is the speed of light.

Fig. $5 \mathrm{a}$ displays the phase angle response, measured at $300 \mathrm{kHz}$ ( $\Omega=1.88 \times 10^{6} \mathrm{rad} / \mathrm{s}$ ), for the cavity containing the polymer-coated LPG upon exposure to m-xylene vapour. The concentrations are given as percentages of the saturated vapour concentration value of $10,730 \mathrm{ppm}$. It can be seen that the magnitude of the phase delay increases with increasing $\mathrm{m}$-xylene concentration to about $20 \%$ followed by a decrease in phase delay until about $40 \%$ after which no further change is observed. Although it is apparent that this particular polymer does not recover immediately upon removal of the analyte vapour, the polymer recovers after $90 \mathrm{~min}$ of exposure to nitrogen gas. In previous measurements, the uptake and release 


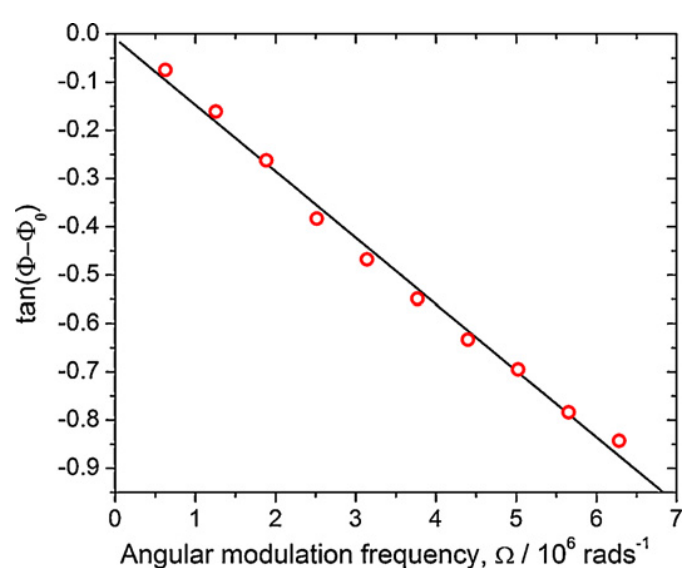

Fig. 4. Determination of the ring-down time of a $6 \mathrm{~m}$ cavity. The solid line is a linear fit (see text for details) and yields $\tau=137 \mathrm{~ns}$.

occurred with a time constant of about 1 min [14]; the slower uptake and release $(5 \mathrm{~min})$ in the present case may be due to a thicker polymer coating.

$\mathrm{m}$-Xylene has a refractive index of 1.4802 , i.e. greater than that of the polymer. Absorption of xylene into the polymer therefore increases the ambient refractive index in the vicinity of the LPG, and shifts the transmission peak to shorter wavelengths. Based on Fig. 3, movement of the LPG transmission peak to shorter wavelengths will first increase and then reduce loss in the optical cavity until a limiting value is reached at which point the LPG peak has moved outside the bandwidth of the FBGs. This occurs at approximately $40 \%$ of the saturation concentration. From the data, the detection limit for xylene vapour is $134 \mathrm{ppm}$ (by partial pressure) or $1.25 \%$ of saturation. The limit of detection is given as $3 \sigma$ of the noise floor of our raw measurement (time constant $1 \mathrm{~s}$ ). If the detector was used to detect $\mathrm{m}$-xylene in the headspace above an aqueous $\mathrm{m}$-xylene solution, the minimum detectable aqueous concentration would be $1.9 \mathrm{ppm}$ by mass. This value is calculated using Henry's law with a constant of $k_{H}=0.13 \mathrm{M} / \mathrm{atm}$ for m-xylene in water [19].

Trichloroethylene (TCE) has a refractive index of 1.4774. Since the refractive index of TCE is greater than that of the polymer, the vapour response curve has qualitatively the same shapes as that of xylene. The saturated vapour concentration value is $77,952 \mathrm{ppm}$, resulting in an increase of phase delay until about $20 \%$, followed by a decrease of phase delay until about $70 \%$, after which no further changes are observed (Fig. 5b). The detection limit for TCE vapour is $3300 \mathrm{ppm}$ or $4.1 \%$ of saturation, corresponding to a TCE concentration of $44 \mathrm{ppm}$ by mass in aqueous solution $\left(k_{H}=0.1 \mathrm{M} / \mathrm{atm}\right)$ [19] when detection was in the headspace.

The concentration dependent responses of the sensor to cyclohexane and gasoline vapour are shown in Fig. 5c and d. Cyclohexane has a much higher saturation vapour pressure $(111,000 \mathrm{ppm}) \mathrm{com}-$ pared to m-xylene. The refractive index of cyclohexane is 1.4141 at $1550 \mathrm{~nm}$, i.e. lower than that of the polymer. The refractive index of gasoline is about 1.424 [20] with a saturation vapour concentration estimated at $355,000 \mathrm{ppm}$. The LPG peak shifts to longer wavelengths upon absorption of either vapour, consistent with an uptake model in which the volume of the polymer expands by an amount corresponding to the volume of the liquid organic compound. As a result of the placement of the LPG transmission peak with respect to the FBG peak, the limiting response occurs at approximately $15 \%$ saturation for both cyclohexane and gasoline (Fig. $5 \mathrm{c}$ and $\mathrm{d}$ ). The

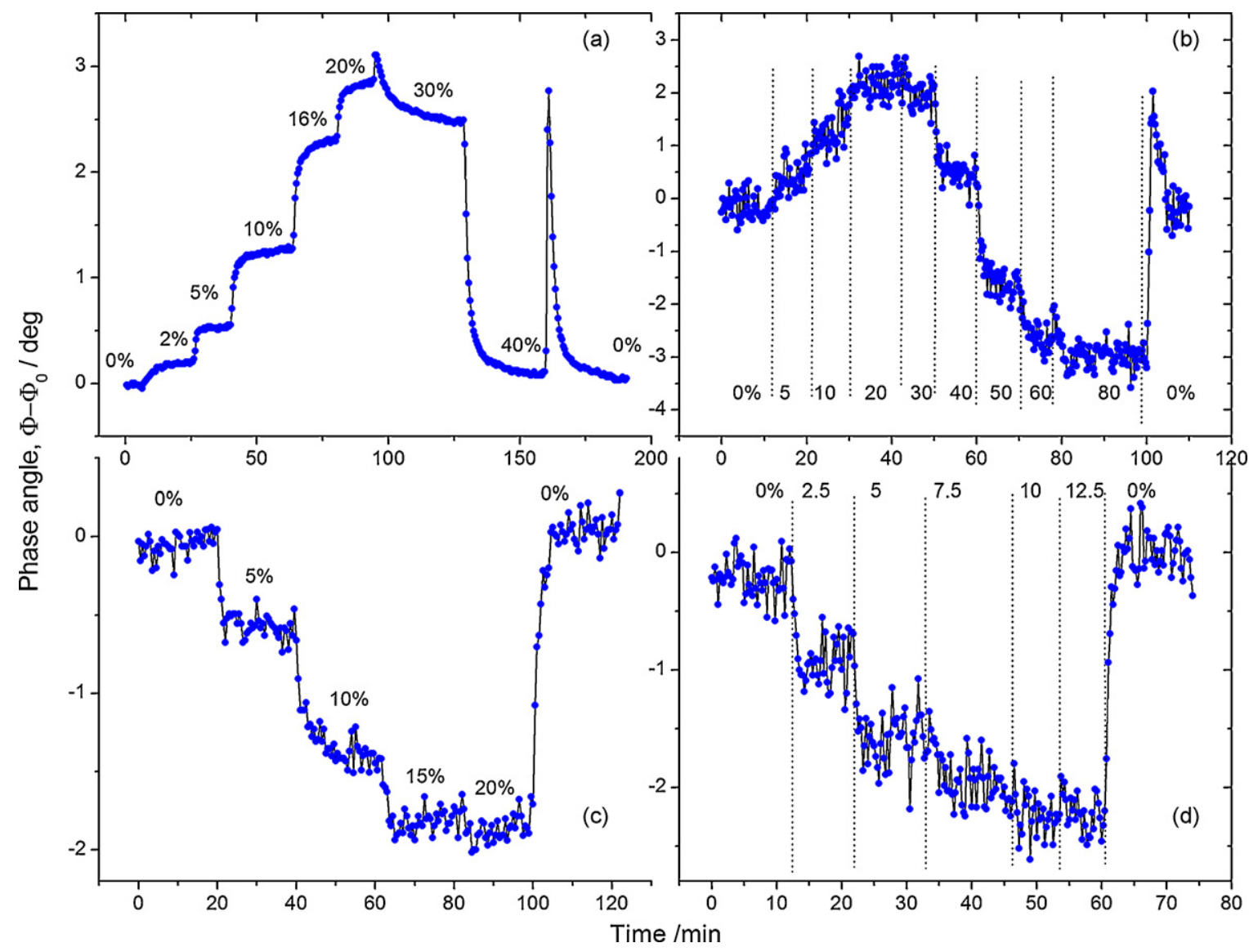

Fig. 5. Response of the etched LPG in a FBG cavity to (a) m-xylene, (b) trichloroethylene, (c) cyclohexane, and (d) gasoline vapour exposures when coated with a PMOS-PDMS polymer of refractive index 1.428 . The concentrations are given with respect to percent of the saturation pressure. 
detection limit for cyclohexane is $1850 \mathrm{ppm}$ or $1.7 \%$ of saturation and $10,500 \mathrm{ppm}$ (3.0\% saturation) for gasoline.

Given the much lower vapour pressure of m-xylene relative to the other three volatile solvents, it is obvious that the methyloctyl PDMS polymer has a much greater affinity for m-xylene than for cyclohexane, TCE, and gasoline. Indeed, our measurements of the polymer/water partition coefficients indicate that $\mathrm{p} K_{\mathrm{pw}}(\mathrm{m}$ xylene $)=3.76, \mathrm{p} K_{\mathrm{pw}}(\mathrm{c}$-hexane $)=2.87$, and $\mathrm{p} K_{\mathrm{pw}}(\mathrm{TCE})=2.93$ [16]. It is also apparent that the sensitivity to gasoline vapour is limited by the similar RI values of the polymer compared to the gas mixture sampled in the headspace of the gasoline liquid. Of course, only a very small change of RI is expected when the liquid that is absorbed into the polymer has a similar RI to the polymer matrix itself.

The noise associated with the measurements (Fig. 5) is notably lower for m-xylene than the other analytes, and is highest for gasoline. This is likely also related to the relative analyte RI and partition factors. Analytes like m-xylene with relatively low vapour pressure and a RI quite different from the PDMS film will produce a given signal with a small amount of analyte in the polymer. Analytes like TCE and cyclohexane, with RI closer to that of PDMS, will require more analyte in the film for a given signal, as will hydrocarbons like those found in gasoline. The differences seen in the noise along with the reduced sensitivity (on a ppm partial pressure scale) are probably a result of increased swelling when a greater quantity of analyte is in the polymer. It is also possible that differences in the vapour mixing system could occur for higher vapour pressure analytes, and especially for mixtures of hydrocarbons as in gasoline.

We attempted to detect the same organic compounds directly in aqueous solution by submersing the coated LPG, but were unsuccessful. The PDMS/PMOS polymer, although widely considered hydrophobic, nevertheless absorbed a considerable amount of water and the LPG attenuation feature moved out of the cavity detection window. We attempted to increase the PDMS/PMOS surface hydrophobicity by reacting the polymer coating with trimethylchlorosilane vapour-a process that was expected to decrease the number of available hydroxyl-terminated sites. Nevertheless, the direct detection of organic contaminants in aqueous solution was overshadowed by water uptake.

Using the information shown in Figs. 3-5 we can calculate the ring-down time of the cavity at the mid-attenuation point of the LPG as $92 \mathrm{~ns}$ and at the attenuation maximum as $63 \mathrm{~ns}$. A readily detectable phase-shift of $0.2^{\circ}$ ( $2 \% \mathrm{~m}$-xylene vapour, Fig. 4 ) then corresponds to a 2 ns decrease of the cavity ring-down time. From the slope of the LPG peak at the mid-attenuation point $(0.015 \mathrm{~dB} / \mathrm{nm})$ we can furthermore calculate that the peak maximum has shifted by only $2 \mathrm{~nm}$ upon uptake of $2 \% \mathrm{~m}$-xylene vapour. Such a peak shift may be resolved using an optical spectrum analyzer [14] or using a laser frequency locking scheme, such as the Pound-Drever-Hall method. Both of these measurements are complicated by the peak's large full-width half-maximum of about $60 \mathrm{~nm}$ (Fig. 3) and are considerably more expensive compared to the phase-shift CRD method.

\section{Conclusion}

We used a long-period grating as a refractive index sensor in combination with solid-phase microextraction for chemical selection and enrichment, and cavity ring-down detection in a waveguide cavity for signal amplification. Using CRD the optical loss is amplified by a factor of 4.7 compared to a single pass measurement. In addition, all CRD measurements are immune to variations in laser power or to detector drifts, since the signal is extracted from an intensity-rate measurement.

All fiber optic sensors for combustible vapours have the advantage that they do not pose an explosion risk due to sparks. The sensor demonstrated a fast response ( $5 \mathrm{~min}$ ), rudimentary chemical selectivity, and low detection limit to four different volatile solvent vapours. In a previous study we incorporated a similarly coated LPG into a waveguide cavity that consisted of a passive loop [14]. The present sensor exhibits an improved detection limit, possibly due to the lower roundtrip loss; we used two fiber/fiber couplers in the previous setup but only one in the present experiment. Also the present sensor is ultra-compact and lends itself to "dipstick" applications, since the sensing element is placed near the end of the fiber strand. It is straightforward to install the sensor head in very confined spaces.

On the other hand, the FBG cavity operated in only a very limited wavelength range and it was difficult to prepare the LPG, by etching and coating, in such a way that the mid-attenuation point of the desired attenuation peak fell into this cavity range. To overcome this complication, FBGs, which exhibit a wider reflection band, may be used. Alternatively a broad band linear fiber cavity may be formed by gold-coating the fiber end faces-as was demonstrated by von Lerber et al. [21,22]. Of course, the cost of the system may be reduced considerably by replacing the combination of a broadly tunable diode laser and EDFA with a fixed-wavelength laser diode of higher output power. This has not been practical in the present exploratory work, since we would then require a different laser diode for every FBG cavity that we used. Also the expensive lock-in amplifier may be readily replaced by phase comparator integrated circuits as was demonstrated previously [14].

Finally, the detection limit may be further decreased and the sensitivity increased by using coating materials that exhibit a higher affinity to the compounds of interest. In a very recent study by Zhang et al. zeolites were shown to have an excellent response to isopropanol and toluene and there is good reason to assume that in our system zeolites would also perform better than the PDMS/PMOS co-polymer [23].

\section{Acknowledgements}

This work was supported by the Natural Sciences and Engineering Research Council of Canada. We thank ITF Labs for advice on fiber optic gratings and for providing the long-period gratings used in this work.

\section{References}

[1] V. Bhatia, Applications of long-period gratings to single and multi-parameter sensing, Opt. Exp. 4 (1999) 457.

[2] S.W. James, R.P. Tatam, Optical fibre long-period grating sensors: characteristics and application, Meas. Sci. Technol. 14 (2003) R49-R61.

[3] V. Bhatia, A.M. Vengsarkar, Optical fiber long-period grating sensors, Opt. Lett. 21 (1996) 692.

[4] B.H. Lee, Y. Liu, S.B. Lee, S.S. Choi, J.N. Jang, Displacements of the resonant peaks of a long-period fiber grating induced by a change of ambient refractive index, Opt. Lett. 22 (1997) 1769.

[5] K.S. Chiang, Y.Q. Liu, M.N. Ng, X.Y. Dong, Analysis of etched long-period fibre grating and its response to external refractive index, Electron. Lett. 36 (2000) 966.

[6] P. Pilla, P.F. Manzillo, V. Malachovska, A. Buosciolo, S. Campopiano, A. Cutolo, L. Ambrosio, M. Giordano, A. Cusano, Long period grating working in transition mode as promising technological platform for label-free biosensing, Opt. Exp. 17 (2009) 20039.

[7] D.W. Kim, Y. Zhang, K.L. Cooper, A. Wang, Fibre-optic interferometric immunosensor using long period grating, Electron. Lett. 42 (2006) 324.

[8] M.P. DeLisa, Z. Zhang, M. Shiloach, S. Pilevar, C.C. Davis, J.S. Sirkis, W.E. Bentley, Evanescent wave long period fiber Bragg grating as an immobilized antibody biosensor, Anal. Chem. 72 (2000) 2895.

[9] J. Zhang, X.L. Tang, J.H. Dong, T. Wei, H. Xiao, Zeolite thin film-coated long period fiber grating sensor for measuring trace organic vapors, Sens. Actuators B: Chem. 135 (2009) 420.

[10] H.J. Patrick, A.D. Kersey, F. Bucholtz, Analysis of the response of long period fiber gratings to external index of refraction, J. Lightwave Technol. 16 (1998) 1606.

[11] R.S. Brown, I. Kozin, Z. Tong, R.D. Oleschuk, H.-P. Loock, Fiber-loop ring-down spectroscopy, J. Chem. Phys. 117 (2002) 10444. 
[12] A. O'Keefe, D. Deacon, Cavity ring-down optical spectrometer for absorption measurements using pulsed laser sources, Rev. Sci. Instrum. 59 (1988) 2544

[13] Z.G. Tong, A. Wright, T. McCormick, R.K. Li, R.D. Oleschuk, H.-P. Loock, Phaseshift fiber-loop ring-down spectroscopy, Anal. Chem. 76 (2004) 6594

[14] J. Barnes, M. Dreher, K. Plett, R.S. Brown, C.M. Crudden, H.P. Loock, Chemical sensor based on a long-period fibre grating modified by a functionalized polydimethylsiloxane coating, Analyst 133 (2008) 1541.

[15] B. Liu, J.H. Luo, G.Y. Kai, Temperature and strain sensor based on weak LPG and fiber ring down, Microw. Opt. Technol. Lett. 50 (2008) 111

[16] K. Plett, Development and Characterization of Polysiloxane Polymer Films for Use in Optical Sensor Technology, Ph.D. Dissertation, Queen's University, 2008.

[17] K. Bescherer, J.A. Barnes, S. Dias, G. Gagliardi, H.P. Loock, N.R. Trefiak, H. Waechter, S. Yam, Measurement of multi-exponential optical decay processes by phase-shift cavity ring-down, Appl. Phys. B 96 (2009) 193.

[18] N.R. Trefiak, Ringdown Spectroscopy in Optical Waveguides, M.Sc. Thesis, Queen's University, 2007.

[19] J. Staudinger, P.V. Roberts, A critical review of Henry's law constants for environmental applications, Crit. Rev. Environ. Sci. Technol. 26 (1996) 205.

[20] R. Falate, R.C. Kamikawachi, M. Muller, H.J. Kalinowski, J.L. Fabris, Fiber optic sensors for hydrocarbon detection, Sens. Actuators B: Chem. 105 (2005) 430.

[21] T. von Lerber, M.W. Sigrist, Cavity ring-down principle for fiber-optic resonators: experimental realization of bending loss and evanescent-field sensing Appl. Opt. 41 (2002) 3567.

[22] D.E. Vogler, M.G. Muller, M.W. Sigrist, Fiber-optic cavity sensing of hydrogen diffusion, Appl. Opt. 42 (2003) 5413.

[23] K.M. Zhou, D. Webb, M. Farries, N. Hayes, L. Zhang, I. Bennion, Biochemical sensor based on a novel all-fibre cavity ring down spectroscopy technique incorporating a tilted fibre Bragg grating, Opt. Laser Eng. 47 (2009) 1023.

\section{Biographies}

Jack A. Barnes is a Senior Research Associate in the Department of Chemistry at Queen's University. His research background includes chemical dynamics, molecular beam laser spectroscopy and the application of spectroscopy to environmental monitoring. His undergraduate degree was obtained at McMaster University, followed by a Ph.D. at the University of Toronto. Postdoctoral work was conducted a the Centre for Molecular Beams and Laser Chemistry at the University of Waterloo. Further work in laser spectroscopy was conducted at the University of Victoria before coming to Queen's University. His current research interests involve the use of fiber optic detectors and microresonator structures in chemical sensing.
R. Stephen Brown completed his B.Sc. in chemistry at Dalhousie University in 1985 He then moved to the University of Toronto, completing his M.Sc. in 1988 and his Ph.D. in 1992 in bioanalytical chemistry. After four years with the National Research Council's Biotechnology Research Institute in Montreal, he joined the Department of Chemistry and the School of Environmental Studies at Queen's University in 1996 where he is now Associate Professor. His group specializes in monitoring water quality, including methods for detecting chemical and microbiological contamination. He co-founded the company Pathogen Detection Systems to commercialize a new bacteria detection technology developed in his laboratory.

Adrienne H. Cheung is completing her final year of undergraduate studies in Biochemistry at Queen's University. She worked as an undergraduate summer student for two consecutive years in the Queen's Department of Chemistry on the development of a fiber optic detector and the use of polymers as microextraction matrices for chemical sensing.

Marian A. Dreher is a research assistant at the Department of Chemistry at Queen's University. He received his Diploma in Chemistry at the University of Konstanz, Germany, in the field of materials science. First introduced to the subject of fiber optic detectors as an exchange student at Queen's University he now works on the development of polymeric coatings for optical platforms designed to detect heavy metals and VOCs.

Gillian Mackey is currently completing a master's degree in the Department of Chemistry at Queen's University. She earned her undergraduate degree in environmental chemistry from Queen's University, completing her honour's thesis with Dr. R. Stephen Brown. Her research interests are in environmental analytical chemistry with a focus on the development of new instruments for contaminated water analysis.

Hans-Peter Loock is an Associate Professor is the Department of Chemistry at Queen's University. His research interests are in the development of instrumentation for analytical spectroscopy, and in laser photochemistry. He obtained his undergraduate degree (Dipl.-Ing.) at the Technical University of Darmstadt, Germany, and completed his doctoral work at the University of Victoria, Canada. After two years of postdoctoral work at the Steacie Institute for Molecular Sciences of the National Research Council of Canada he joined Queen's University, where he has since worked on molecular photodissociation and photoionization dynamics, ice photochemistry, and image analysis. Work on fiber optic detectors includes the development of new absorption detectors and refractive index sensors as well as photoacoustic and mechanical probes. 